\title{
Time-Resolved Emittance Characterization of an Induction Linac Beam Using Optical Transition Radiation
}

G. P. Le Sage

November 5, 2002

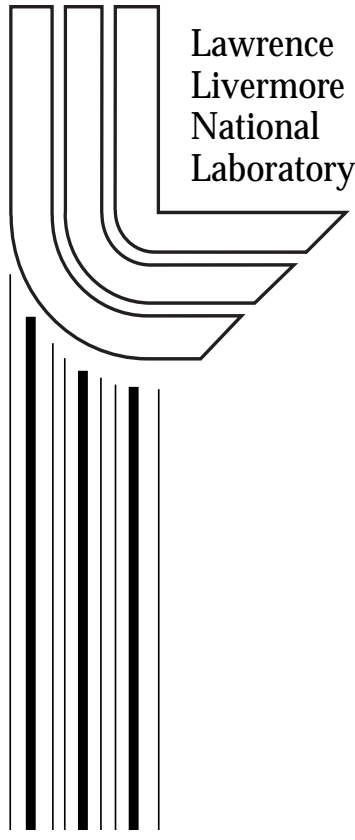




\section{DISCLAIMER}

This document was prepared as an account of work sponsored by an agency of the United States Government. Neither the United States Government nor the University of California nor any of their employees, makes any warranty, express or implied, or assumes any legal liability or responsibility for the accuracy, completeness, or usefulness of any information, apparatus, product, or process disclosed, or represents that its use would not infringe privately owned rights. Reference herein to any specific commercial product, process, or service by trade name, trademark, manufacturer, or otherwise, does not necessarily constitute or imply its endorsement, recommendation, or favoring by the United States Government or the University of California. The views and opinions of authors expressed herein do not necessarily state or reflect those of the United States Government or the University of California, and shall not be used for advertising or product endorsement purposes.

This work was performed under the auspices of the U. S. Department of Energy by the University of California, Lawrence Livermore National Laboratory under Contract No. W-7405-Eng-48.

This report has been reproduced directly from the best available copy.

Available electronically at http://www.doc.gov/bridge

Available for a processing fee to U.S. Department of Energy

And its contractors in paper from

U.S. Department of Energy

Office of Scientific and Technical Information

P.O. Box 62

Oak Ridge, TN 37831-0062

Telephone: (865) 576-8401

Facsimile: (865) 576-5728

E-mail: reports@adonis.osti.gov

Available for sale to the public from

U.S. Department of Commerce

National Technical Information Service

5285 Port Royal Road

Springfield, VA 22161

Telephone: (800) 553-6847

Facsimile: (703) 605-6900

E-mail: orders@ntis.fedworld.gov

Online ordering: http://www.ntis.gov/ordering.htm

OR

Lawrence Livermore National Laboratory

Technical Information Department's Digital Library

http://www.llnl.gov/tid/Library.html 


\title{
Time-resolved emittance characterization of an induction linac beam using Optical Transition Radiation
}

\author{
G. P. Le Sage \\ Lawrence Livermore National Laboratory, Livermore, CA
}

\begin{abstract}
An induction linac is used by Lawrence Livermore National Laboratory to perform radiographic testing at the Flash X-ray Radiography facility. Emittance characterization is important since $\mathrm{x}$-ray spot size impacts the resolution of shadow-graphs. Due to the long pulse length, high current, and beam energy, emittance measurement using Optical Transition Radiation is an attractive alternative for reasons that will be described in the text. The utility of OTR-based emittance measurement has been well demonstrated for both RF and induction linacs. We describe the time-resolved emittance characterization of an induction linac electron beam. We have refined the optical collection system for the induction linac application, and have demonstrated a new technique for probing the divergence of a subset of the beam profile. The experimental apparatus, data reduction, and conlusions will be presented. Additionally, a new scheme for characterizing the correlation between beam divergence and spatial coordinates within the beam profile will be described.
\end{abstract}

PACS Codes: 29.27.-a, 41.75.Fr, 41.75.Ht

\section{INTRODUCTION}

Induction linac-based radiography plays an important role in measuring the dynamic properties of dense materials. The LLNL Flash X-Ray Radiography (FXR) facility is capable of producing high doses of x-ray radiation using a $3 \mathrm{kA}, 70 \mathrm{~ns}, 17.5 \mathrm{MeV}$ electron pulse focused into a Tantalum Bremsstrahlung target [1]. The most important quality factors of the x-ray probe beam produced at FXR are spot size and dose. The xray spot size determines the spatial resolution of an x-ray shadow-graph or radiograph. The x-ray spot size is determined by the focused electron beam radius at the $\mathrm{x}$-ray conversion target. Electron beam radius is determined by four main factors: beam emittance, energy spread, beam motion, and focusing aberrations [2]. Emittance is a measure of the phase-space volume of an electron beam. At a beam waist, the correlation between particle position and angle is minimized. The remaining random angular spread of the beam, together with the radial profile characterizes transverse beam emittance [3]. The beam emittance, multiplied by $\beta \cdot \gamma$, the normalized velocity and the relativistic Lorentz factor, is an invariant quantity. While the total normalized phase-space volume is constant, acceleration and transport forces with nonlinear radial profiles can degrade the effective transverse emittance of a beam, defined by drawing a contour around a given percentage of particles in phase space. Normalized beam divergence that is uncorrelated to transverse or longitudinal coordinates in the beam cannot be later reduced. By measuring the beam divergence angle and radius at a waist, we can characterize the beam emittance, and expect the measured value to contribute to the final spot radius at the x-ray production target. Emittance measurement is a tool for optimization in the sense that the beam injector settings can be tuned for a minimum 
emittance, and the acceleration and transport settings can be tuned to produce an effective normalized emittance value that is not increasing.

Emittance can be characterized using several different conventional techniques. These include collimation of the beam with small apertures ("pepper-pot" characterization) [4], magnet scan techniques where the beam radius is characterized as a function of focusing parameters [5], and multi-profile techniques where the beam envelope is characterized simultaneously at several points [6]. Using Optical Transition Radiation (OTR) to characterize electron beam emittance works in a fundamentally different way in that the beam divergence and emittance are characterized at a single point in a single shot, while the envelope is not scanned to make a measurement. A charged particle produces OTR when it crosses a boundary between two media with different dielectric properties. One example is an electron passing through a thin metallic foil under vacuum. This light is emitted with an angular distribution that depends on the particle energy and incident angle. For relativistic electron beams, measurable transition radiation light is emitted across the visible range. Using OTR, collection of a single optical pattern gives the divergence of an electron beam. A theoretical OTR pattern can be generated mathematically, using a prescribed beam divergence profile, and fit to measured data. With beam divergence characterized, combination with spatial profile information gives the beam emittance. The OTR diagnostic provides a means to measure both spatial and angular profiles of an electron beam.

The first theoretical treatment of transition radiation was published in 1946 [7]. Beam characterization using OTR has been utilized from 1975 through the present [8-11]. More recently, OTR has been used to characterize long-pulse, high-current beams produced by induction linacs [12]. The technique continues in refinement, and here we report emittance measurement results for the FXR beam using OTR and propose a new method to characterize the correlation between beam divergence and spatial coordinates within the beam profile.

A few definitions are required to introduce the OTR measurement technique. Refer to Fig. 1 for the following description. In three-dimensional Cartesian space, we will consider an electron incident from the $-\mathrm{X}$ direction. The foil is oriented along the $\mathrm{Z}$ direction with its surface normal at $-45^{\circ}$ in the $\mathrm{X}-\mathrm{Y}$ plane. The specular direction, corresponding to the direction that light would reflect from the foil if that light were incident in the same direction as the electron, coincides with the $\mathrm{Y}$ axis for an electron incident from the $-\mathrm{X}$ direction $\left(90^{\circ}\right.$ reflection). The $\mathrm{X}-\mathrm{Y}$ plane will be called the "horizontal" plane, and the Y-Z plane the "vertical" plane. 


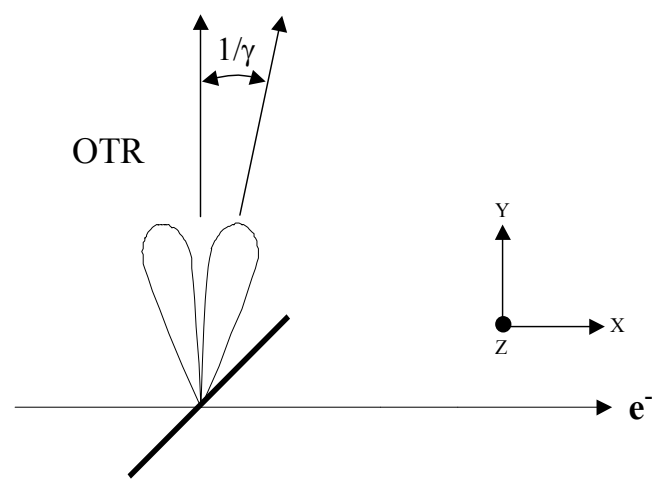

Figure 1: Schematic of the OTR foil

The OTR produced by a relativistic electron beam impinging on a foil target is characterized by a cone of light peaked at an angle of $1 / \gamma$. The center of the pattern coincides with the specular direction. The OTR light is radially polarized, with the polarization vector drawn between the specular direction and the direction of the emitted OTR photon. An electron beam, which is a collection of particles with different energies, positions, and angles produces a "blurred" OTR pattern based on the summation of the patterns produced by the individual electrons. Figure 2 shows a lineout through the center of an OTR pattern produced by a $17.5 \mathrm{MeV}$ electron. The two curves show a single electron OTR pattern (red), and a "blurred" pattern (blue) produced by a distribution of angles with a standard deviation of 15 milliradians $(\mathrm{mr})$. The light pattern is plotted as a function of the observation angle multiplied by the Lorentz relativistic factor $\gamma$.

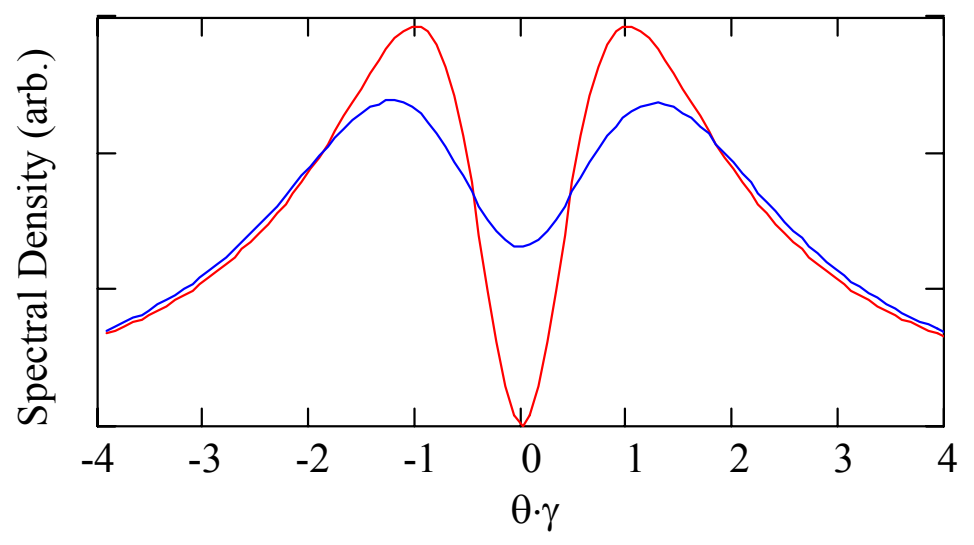

Figure 2: Vertical plane OTR lineouts for a single electron and for $15 \mathrm{mr}$ divergence

By using a linear polarizer and taking a lineout of the OTR pattern through the center null, the divergence of the electron beam in that polarization direction can be characterized. We utilized a linear polarizer for most of our beam characterization measurements, and also typically measured the OTR light emitted in the Y-Z or "vertical" plane as previously defined. The vertical plane was selected for several key 
reasons. For spatial analysis, depth of focus is not an issue if the spatial profile is analyzed with a vertical lineout. For an object close to the optical collection system, magnification can change in a horizontal scan across a tilted foil. That problem is eliminated in the vertical scan direction. Last, a symmetrical OTR pattern model can be applied for analysis of the angular profile. In the horizontal plane, one must consider the angular dependence of the Fresnel reflection coefficients for a given material at a given wavelength since they produce an asymmetric OTR pattern. For vertically polarized OTR measurements, the overall light output will scale with a given foil angle, material, and wavelength, but the OTR lobes will remain symmetrical. For relativistic electron beams, the expression for the intensity of OTR light produced parallel to the plane of observation using the small angle forms of $\sin \theta$ and $\cos \theta$ is given by the expression [10]

$$
\frac{\partial^{2} I_{\|}}{\partial \omega \partial \Omega}=\frac{e^{2}}{c} \frac{\beta^{2}}{\pi^{2}} \frac{\left|r_{\|}\right|^{2} \theta^{2}}{\left(\gamma^{-2}+\theta^{2}\right)^{2}}+\frac{\operatorname{Re}\left(r_{\|}\right) \theta_{x}}{\gamma^{-2}+\theta^{2}},
$$

where $r_{\|}$is the Fresnel reflection coefficient in the horizontal plane, $\theta$ is the observation angle with respect to the specular direction, $\theta_{\mathrm{x}}$ is the observation angle measured in the horizontal plane, $\omega$ is the frequency of the observed light, and $\Omega$ is the angle in steradians. For the horizontally polarized OTR pattern, the second term in Eq. 1 gives an asymmetry as $\theta_{\mathrm{x}}$ changes sign. The vertically polarized pattern will show no lobe asymmetry as shown in Fig. 2. The horizontally polarized OTR patterns for a single electron are shown in Fig. 3. The examples shown account for the reflection coefficients of Aluminum and Kapton ${ }^{\circledR}$ at a specular angle of $45^{\circ}$ at a $500 \mathrm{~nm}$ wavelength [13 - 15]. The Kapton pattern has been scaled by a factor of 21.5 to match peak intensity with Aluminum. The differences in the polarization-dependent reflection characteristics of various materials were used to advantage in demonstration of a new measurement technique described in a later section.

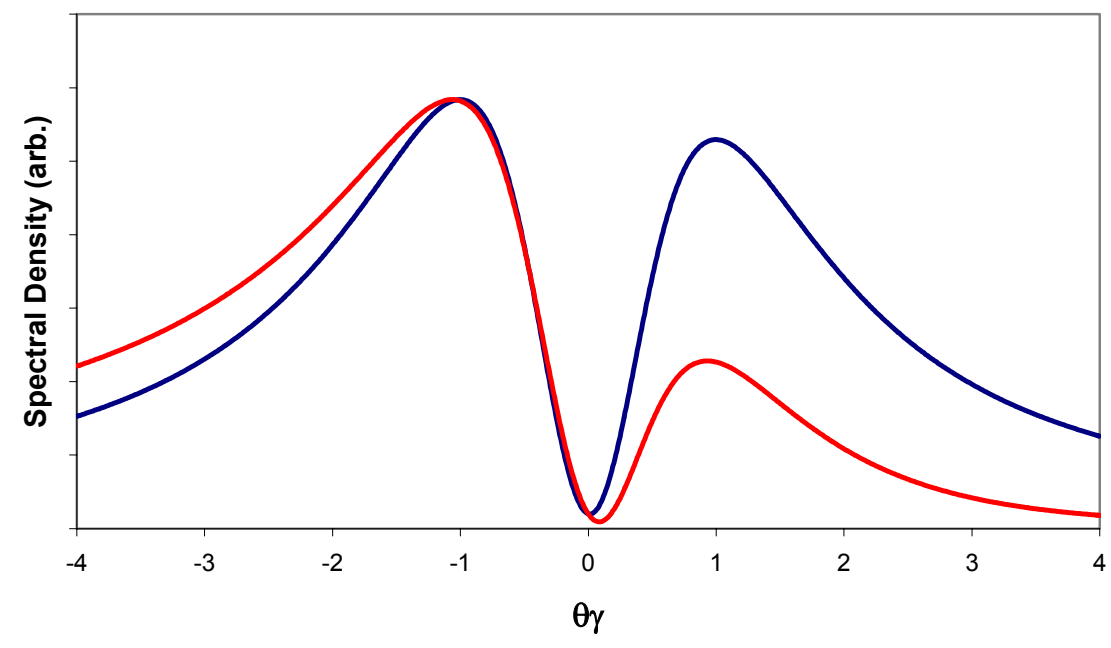


Figure 3: Ideal OTR pattern at $17.5 \mathrm{MeV}$, horizontal polarization, Aluminum and Kapton foil targets with $45^{\circ}$ tilt angle

The essential components of an OTR beam diagnostic system are the target, which in our case is an $7.6 \mu \mathrm{m}$ thick aluminized KAPTON foil, an optical system for collecting the divergent OTR light pattern, and a detector that can collect and record the angular distribution of OTR light. Figure 4 shows two schematic configurations, the first used to collect OTR light in order to record the angular divergence of the incident electron beam, and second configured to collect the spatial profile of the beam. Representing the optical collection system by a single lens, the angular collection scheme sets the distance from the detector exactly equal to the focal length of the lens. In that scheme, incident angles are mapped directly to positions on the detector through the relationship $R_{\text {detector }}=\theta_{\text {incident }}$ $f$. The spatial collection system places the detector at a distance of $2 \cdot f$ from the lens, resulting in point-to-point spatial imaging.
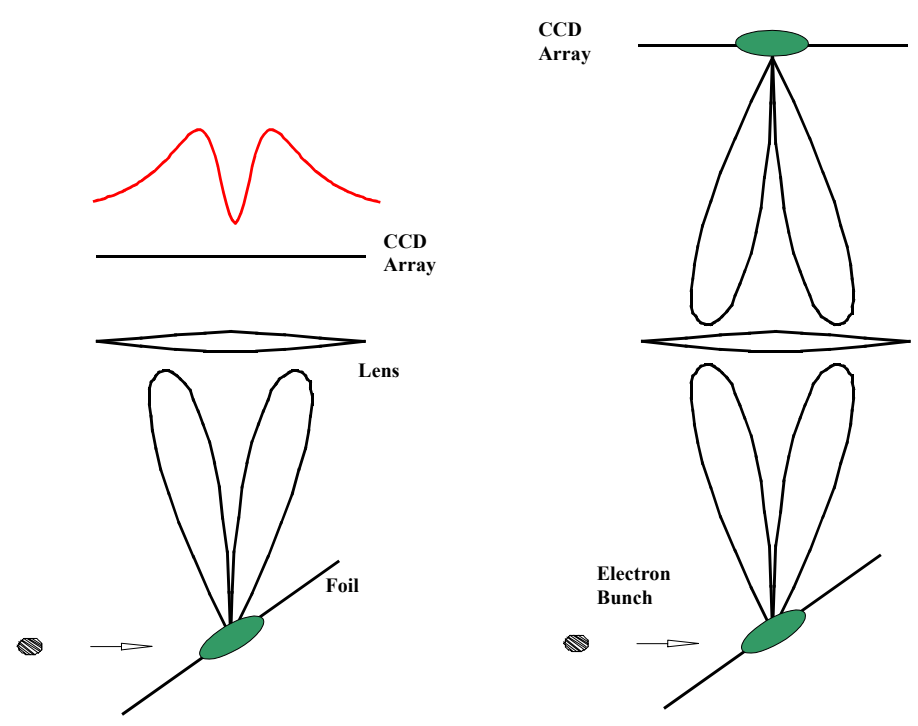

Figure 4: OTR collection of divergence and spatial profile

In the next two sections, we describe the experimental arrangement used to collect OTR data from the FXR beam and data reduction. A new technique for characterizing the correlation between beam divergence and spatial coordinates within the beam profile will then be presented with a description of the first demonstration experiment. Lastly, conclusions and future plans will be discussed.

\section{EXPERIMENTAL ARRANGEMENT}

Considering the high current, beam energy, and long pulse length produced by the FXR linac, our first consideration in selecting OTR as a beam diagnostic was foil damage caused by beam-induced heating. For a relativistic electron beam, the beam energy deposited in a target scales linearly with thickness. The temperature increase of the foil scales with volume, which is also linear with respect to thickness. The overall foil 
heating resulting from interaction with an electron beam is therefore independent of foil thickness. Conduction and radiative cooling can be ignored since the deposited power is orders of magnitude greater than power dissipation, at least on the time-scale of the beam interaction $(70 \mathrm{~ns})$, so we considered the foil heating to be instantaneous. We calculated the temperature increase for the aluminized Kapton foil during one shot of the FXR beam. Given the melting point of Aluminum and Kapton, we were able to determine the minimum transverse beam radius that would not melt the foil target. We calculated that thin dielectric and metallic foils could tolerate a charge density of $2-3 \mu \mathrm{C} / \mathrm{mm}^{2}$ at the FXR beam energy. With a beam radius of $5 \mathrm{~mm}$, the single shot charge density is 2.45 $\mu \mathrm{C} / \mathrm{mm}^{2}$ for a $70 \mathrm{~ns}, 3 \mathrm{kA}, 17.5 \mathrm{MeV}$ beam $(224 \mu \mathrm{C}$ per shot), assuming a uniform profile. In practice, we found that this calculated limit corresponded closely to the actual damage threshold. With freedom to choose any target thickness, we chose a thin foil target with a thickness of $7.6 \mu \mathrm{m}$. This thin, aluminized foil could be easily stretched flat in an appropriate holder, and had an additional advantage of minimally scattering the electron beam. With 7.6 $\mu \mathrm{m}$ of Kapton and 300 Angstroms of Aluminum, the scattering angle for a $17.5 \mathrm{MeV}$ beam is $3.65 \mathrm{mr}$ [16], which adds in quadrature with the incident beam divergence. The beam can be easily transported after the OTR measurement to an appropriate beam dump or other target. The OTR foil target stops a very small fraction of the total beam current.

Measurement of divergence based on OTR is limited to a range between a low divergence beam that becomes indistinguishable from the single electron pattern, and a high divergence beam where the peak and null features of the OTR pattern become completely washed out. The details of this divergence range are discussed in the data reduction section. For single foil OTR measurements, the useful diagnostic range will later be demonstrated to be limited between approximately $26 \%$ and $95 \%$ of $1 / \gamma$. This range sets the required beam radius for a given emittance value. Divergence values of several times $1 / \gamma$ can also be measured using single foil OTR by fitting to the width and height of a single peak when the central null is filled in, but in this analysis, we relied on fitting to the peak and null values, and normalized each data set. We expected to measure a normalized emittance of approximately $300 \mathrm{~cm}-\mathrm{mr}$, corresponding to a beam divergence of approximately $17 \mathrm{mr}$ or $60 \%$ of $1 / \gamma$ for a $5 \mathrm{~mm}$ beam radius. Since this divergence represents the upper bound of our expected measurements, single foil OTR was well suited to divergence characterization for the FXR beam.

A CCD pixel array of 512 x 512 elements and a size of $13.25 \mathrm{~mm}$ square was used to record both the angular and spatial patterns of OTR light produced by the foil target. A gated optical intensifier (GOI) was used to allow measurement of temporal slices down to the $1 / 2 \mathrm{~ns}$ range. The GOI comprises a photocathode, a micro channel plate (MCP), and an electrical pulsing system. The GOI has an equivalent 7-8 bit dynamic range based on a CCD noise floor of approximately 50 counts, and a nonlinear response above approximately 350 counts. The CCD was linked to the optical collection system through a fiber bundle. The fiber bundle is made of $400 \times 400$ strands of $10 \mu \mathrm{m}$ diameter. The measured resolution of the CCD / GOI / fiber bundle combination was 6.5 line pairs per $\mathrm{mm}$ at $50 \%$ of peak system gain at the fiber bundle interface. One challenge in the 
experiment was designing an optical collection system that could match or exceed the limiting resolution of the CCD / GOI / fiber combination.

In order to accurately compare measured OTR data to theoretically generated patterns, a wide angular range needs to be recorded. We collected an angular range of $\pm 4 / \gamma$, corresponding to $\pm 6.5^{\circ}$ from the source point. One must also account for the finite beam radius when designing the optical system since the full optical range must be collected from every point in the beam profile so as not to add a spatial convolution to the resulting measured data. In order to collect the full range of OTR light, we based our optical collection system on two 8-inch diameter lenses, with the first lens only 11.5 inches from the OTR target foil. For the angular collection system, an optics package consisting of two additional doublet lenses was used to correct for spherical aberration generated by the large plano-convex lenses. The lens system was designed using the OSLO ${ }^{\circledR}$ computer code. The optics arrangement for the angular collection of OTR light is shown schematically in Fig. 5.

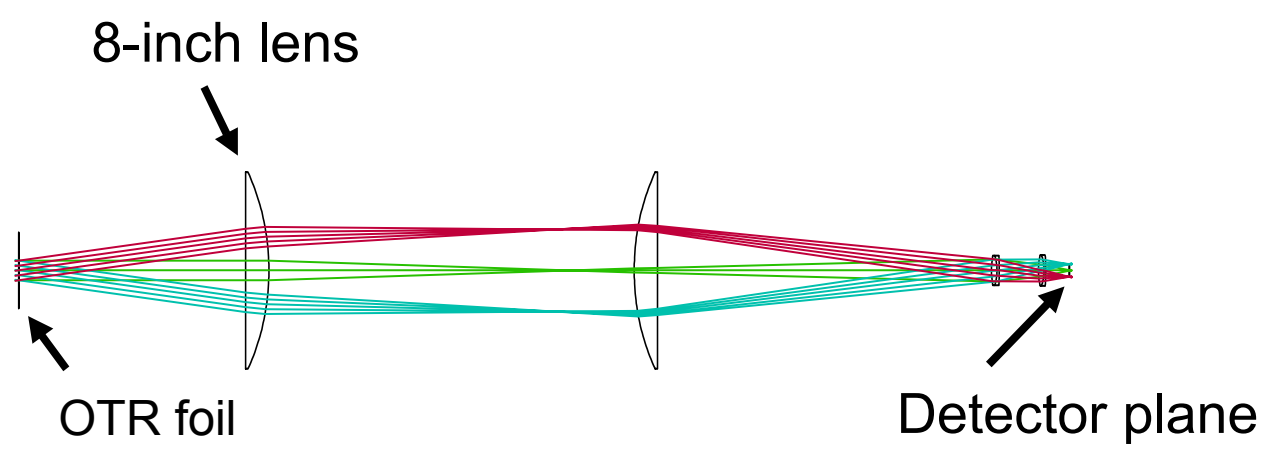

Figure 5: Optics arrangement for collection of OTR angular pattern

The colors shown in Fig. 5 represent three different light ray angles, the axial rays, and rays launched at $\pm 4 / \gamma$. The rays are launched at several spatial locations across a $10 \mathrm{~mm}$ radius source representing the profile of the incident electron beam. Angular mapping is accomplished by focusing all rays in a given direction on to a single point. In the ideal case, rays launched from any spatial location within the beam profile, and with the same angle are mapped to a single point on the CCD array. All spatial information is lost in this case, and only the angular profile is recorded. An alignment laser launched down the bore of the accelerator near the injector established the specular direction of the OTR light. This step was important since the laser light allowed alignment of the various optics in the system. Additionally, the alignment laser allowed us to perform an angular calibration of the system by making small changes to the tilt angle of the foil target and noting the corresponding spatial location of the light recorded on the CCD.

The performance of the optical system in the angular light collection mode is shown in Fig. 6. Instead of evaluating the effectiveness of imaging a small optical spot to determine the resolution of the system, in this case we evaluate the size of a spot 
generated by launcing parallel rays from the source. The aberrations in the system are characterized by optical spot size collected for a given distance from the optical axis for the ray launched from the source plane. The system we used generates an rms optical spot radius of $53 \mu \mathrm{m}$ at the entrance to the fiber bundle for rays emitted at a radius of 10 $\mathrm{mm}$ from the optical axis over an optical bandwidth from $488 \mathrm{~nm}$ to $656 \mathrm{~nm}$. The mapping of angles to positions on the entrance of the fiber bundle has a scaling of $59 \mu \mathrm{m}$ / mr. Comparing this to the approximate $150 \mu \mathrm{m}$ resolution of the CCD / GOI / fiber combination shows that the optical system is not the limiting factor for angular resolution. Our calibrated angular range of data collection was $8.2 / \gamma$, corresponding to a row of 512 pixels. The angular resolution per pixel was $0.5 \mathrm{mrad}$, but the optical system limited the resolution to approximately $\pm 1.25 \mathrm{mrad}$ or $1.1 \%$ of the total angular range collected. Since the optical collection system was capable of collecting a full angular range of $233 \mathrm{mrad}$, the CCD also represented the limiting aperture for the system, discarding spatial-angular correlations at the outer edges of the angular pattern.

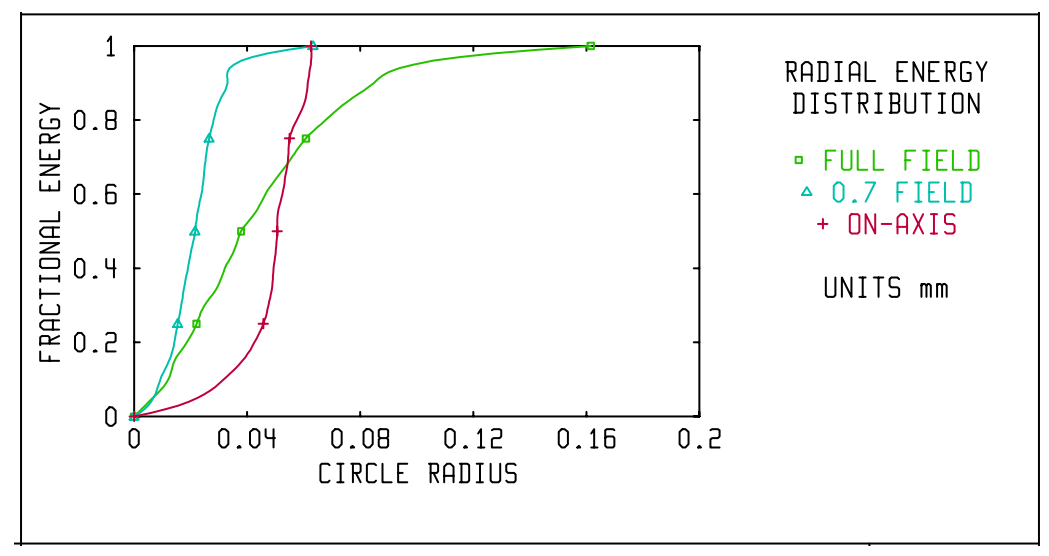

Figure 6: Analysis of angular OTR light collection system using OSLO

An example single foil OTR pattern collected with the above described system is shown in Fig. 7. A linear polarizer was used to select the vertically polarized light emitting from the foil. Recalling that OTR light is radially polarized, and peaked at an angle of $1 / \gamma$, the measured pattern shows the effect of the linear polarizer discarding orthogonal polarization components. Since we calibrated the angular scale, the lobe spacing also verifies $17.5 \mathrm{MeV}$ beam energy. Small angular misalignment of the polarizer was corrected during data analysis by rotating the angular images to align the OTR peaks along the vertical axis. 


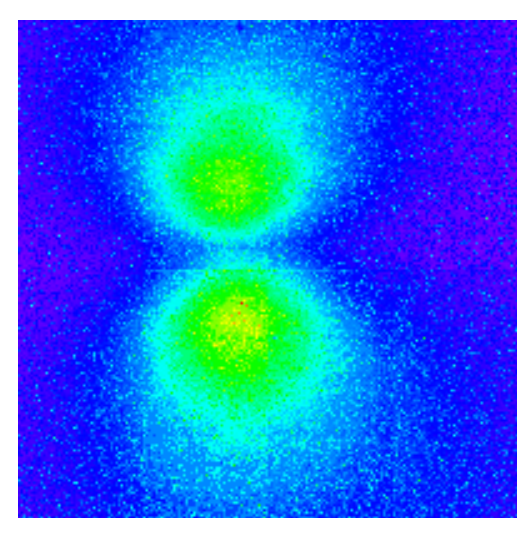

Figure 7: Example OTR angular pattern

An asymmetry can be noted in the lobe peak heights in the figure. Since the figure shows vertical polarization, this asymmetry does not result from the angular dependence of the Fresnel coefficients. In fact the CCD array consists of eight separate regions whose outputs are combined to produce the whole field. The OTR pattern shown spanned across two of these regions. An error in the gain correction between the upper and lower sections caused the apparent asymmetry. For this reason we were required to analyze only half of the OTR pattern. The impact of this limitation is described in the data reduction section.

In order to collect the spatial profile of the electron beam, the optical system was modified with the addition of three lenses to switch to point-to-point spatial imaging. Since the main 8-inch lenses are maintained in their original positions, we were able to remotely switch between angular and spatial profile collection by means of inserting separate optics packages. The combination of both divergence and the spatial profile allows characterization of the beam emittance. The spatial imaging lens package, and the pneumatic system for switching between the angular and spatial imaging packages are shown in Fig. 8.
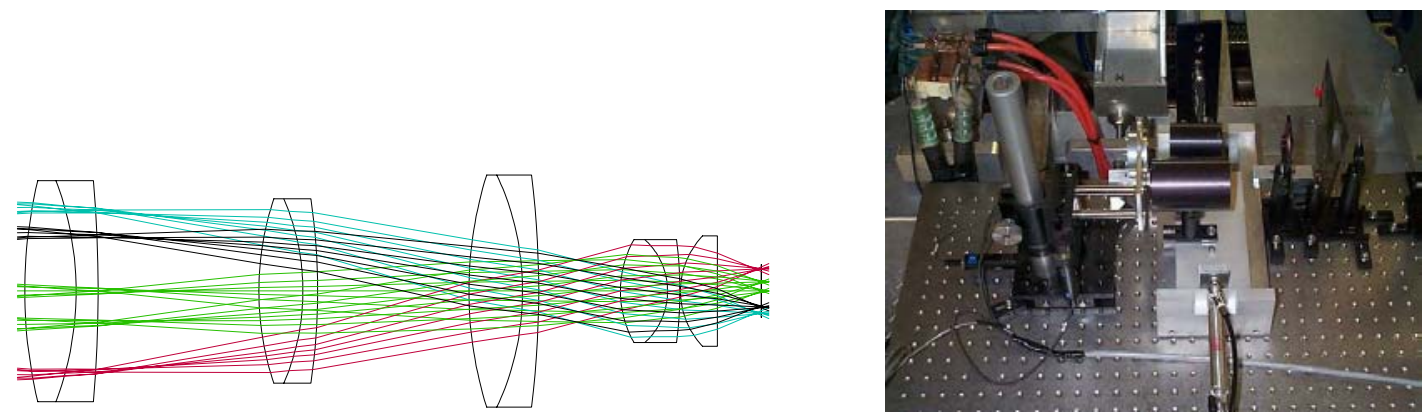

Figure 8: Additional lenses allow collection of beam spatial profile

Optimization and evaluation of the spatial light collection optical system was similarly performed using OSLO. Across a light wavelength band of $488 \mathrm{~nm}$ to $656 \mathrm{~nm}$, the rms optical spot size of the system for a point source at the foil target with a $10 \mathrm{~mm}$ radius was $171 \mu \mathrm{m}$ at the entrance to the fiber bundle. Since the system magnification is 0.31 , 
the rms equivalent optical spot size at the foil target is $552 \mu \mathrm{m}$, or $11 \%$ of a $5 \mathrm{~mm} \mathrm{rms}$ radius. The full field of view for the imaging system was $23.4 \mathrm{~mm}$ corresponding to 374 of 512 pixels across the CCD, with a spatial calibration of $63 \mu \mathrm{m}$ per pixel. The resolution spot radius was added in quadrature to the toelerance of the spatial data fitting to determine the measured tolerance of beam radius. Spatial calibration was performed by imaging objects of known size at the foil target location. An example spatial beam profile collected with this system and a Gaussian fit to a lineout through the image are shown in Fig. 9. A Labview ${ }^{\circledR}$ program was written to automatically process the binary image file, locate the centroid of the image, take a vertical lineout, and perform a leastsquares fit to a Gaussian profile. The program also analyzed the $\chi^{2}$ parameter for the data fitting automatically.
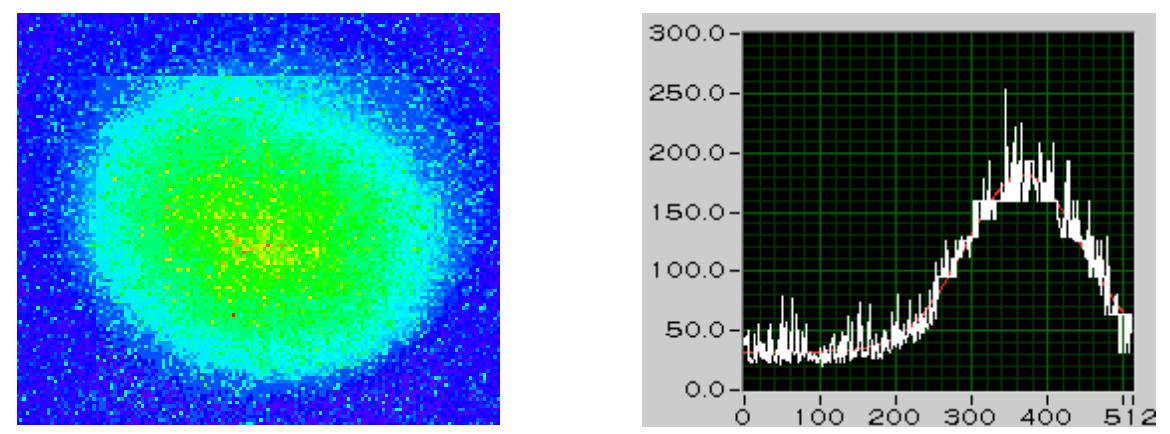

Figure 9: Example spatial profile and lineout fit

The entire optical collection system is shown in Fig. 10. The electron beam was focused to a waist of a prescribed size within the diagnostic vacuum chamber containing the OTR target foil. The optical collection system captured and transmited the angular and spatial profiles into a fiber bundle (not shown in the picture) that attached to a shielding enclosure containing the GOI and CCD. 
Angular / spatial optics

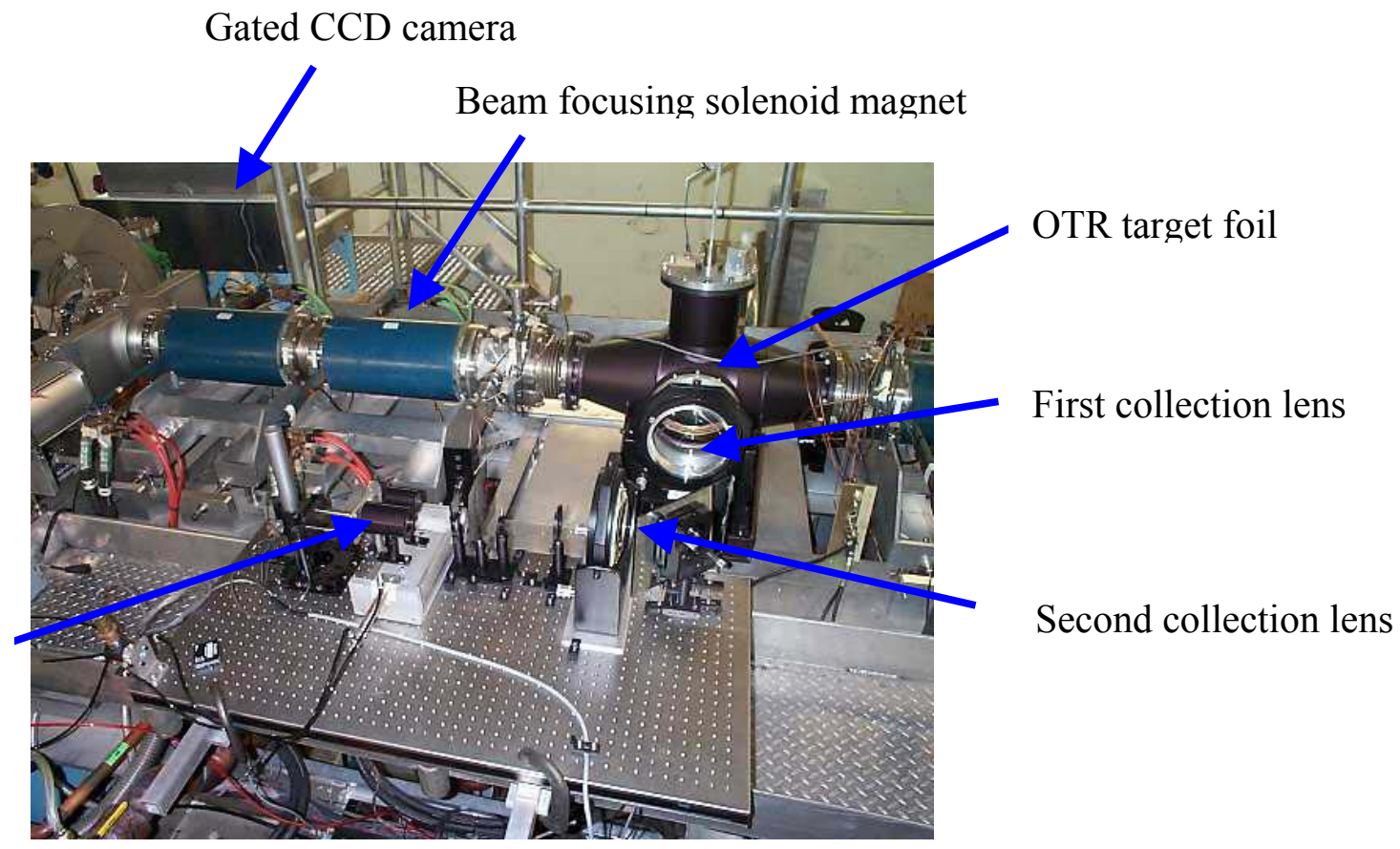

Figure 10: OTR diagnostic arrangement

\section{DATA COLLECTION}

Angular and spatial OTR patterns were collected through the FXR beam pulse at intervals of $10 \mathrm{~ns}$, using a $1 / 2 \mathrm{~ns}$ gate. The beam waist condition was verified through checking transport magnet settings against beam envelope simulations. Our main interest in the series of measurements was both the absolute value and the variation of the beam emittance during the $70 \mathrm{~ns}$ pulse. We sequentially collected spatial profile and OTR angular patterns at each time step. What we measured was a clear and significant variation of both the beam radius and the divergence during the pulse. The angular OTR patterns for the beam for six separate time steps are shown in Fig. 11. The center of the current pulse flat-top corresponds approximately to the $80 \mathrm{~ns}$ gate. Even without numerical analysis, the figure clearly demonstrates a variation in the beam divergence. A corresponding change in the beam radius through the pulse demonstrated that the emittance appeared to remain relatively constant. As the divergence increased, the beam radius decreased. The time-slice emittance characterization is presented in the data reduction section. 


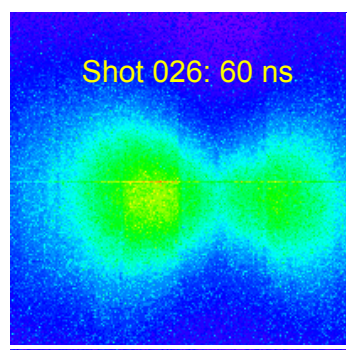

Shot 016: $90 \mathrm{~ns}$

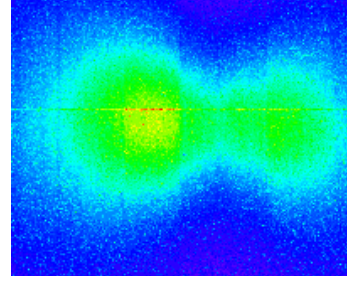

Figure 11: OTR divergence patterns for various time steps through the FXR beam pulse

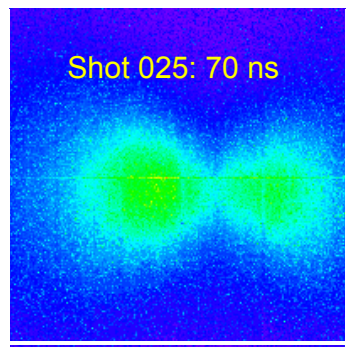

Shot 019: $100 \mathrm{~ns}$

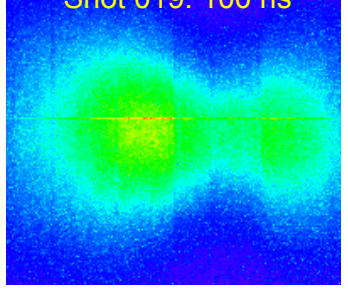

Shot 020: $110 \mathrm{~ns}$
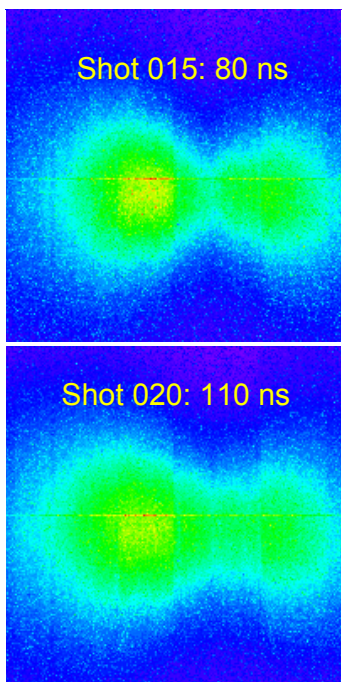

\section{DATA REDUCTION}

The techniques used to analyze angular OTR data are based on comparing normalized, measured data with a theoretical OTR pattern generated mathematically. Since an electron beam is an ensemble of particles with individual positions, directions, and energies, a theoretical OTR pattern can be generated by adding the contributed OTR pattern of individual electrons. This pattern generation has been accomplished using an analytical convolution of the single electron OTR pattern with a normal distribution of particle angles [10], by ray tracing a simulated particle ensemble through an optical collection system model [12], and by using a one-dimensional Monte-Carlo style technique, as in the present analysis. The Monte-Carlo technique starts with a random distribution of particles with normal distributions in space and angle, as shown in Fig. 12. Each electron in this distribution will generate the calculated OTR profile represented in the figure by the red curve at the specular angle of reflection. The distribution of electrons in the beam each produce the single particle OTR pattern in a slightly different direction. When these individual patterns are added together, the "blurred" pattern shown as the blue curve in the figure is produced. The larger the divergence value of the beam, the less sharp the OTR peaks and null become. The small angle form of the OTR profile used in the current analysis is appropriate for electron beams with energy of at least several MeV. For lower beam energy, the exact form of the theoretical OTR profile and the small angle approximation begin to diverge. In the case of low beam energy, the angular range of the OTR lobes also becomes so wide that much of the OTR light may not be collected by a lens and detector system. The effect of limiting the angular field of view is described in the data reduction section. In the case of a low energy beam, the ray tracing technique using the exact form of OTR light production is better suited to analysis of measured OTR profiles [12]. 

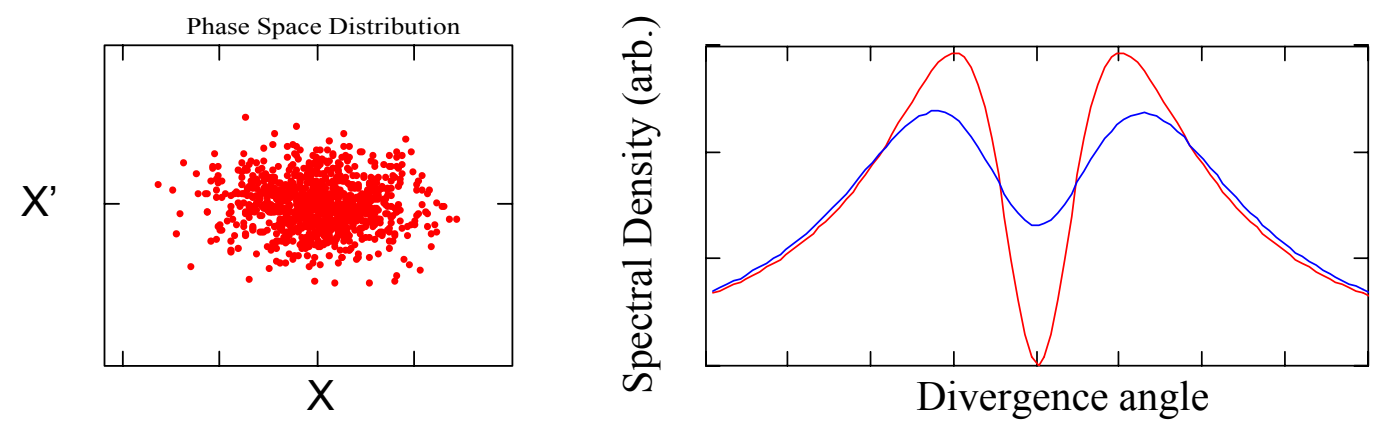

Figure 12: Simulated electron beam and corresponding effect on OTR pattern

The expression used to generate the theoretical OTR patterns for a vertical lineout is given by

$$
\frac{\partial^{2} I_{\|}}{\partial \omega \partial \Omega}=\sum_{m} A \cdot \frac{\left(\theta-\theta_{m}\right)^{2}}{\left(\gamma^{-2}+\left(\theta-\theta_{m}\right)^{2}\right)^{2}}
$$

with $\theta_{\mathrm{m}}$ representing the shift in angle of the OTR light for a single particle in the distribution. The normalized summation of all of the particle contributions gives the theoretical OTR pattern.

For the present analysis of the FXR OTR data, 10,000 particles were generated with a normal distribution using a prescribed rms divergence. The ideal OTR profile was calculated for each simulated particle, and the summation of all of the patterns generated the theoretical OTR patterns used for data fitting. Two example data fits are shown in Fig. 13. As mentioned previously, we were only able to use a single lobe for data fitting due to the artificial lobe asymmetry produced by section gain variations across the CCD. Theoretical OTR patterns for 0 through $40 \mathrm{mr}$ beam divergence in $5 \mathrm{mr}$ steps are shown compared to a measured data profile taken by extracting a lineout through the vertically polarized, measured OTR pattern. For statistical data analysis, $2.5 \mathrm{mr}$ divergence steps were used. The least-squares fit of the two example data sets produced divergence values of $6.3 \pm 0.2$ and $17.3 \pm 0.2 \mathrm{mr}$, as shown in Figs. 13 and 14 respectively.
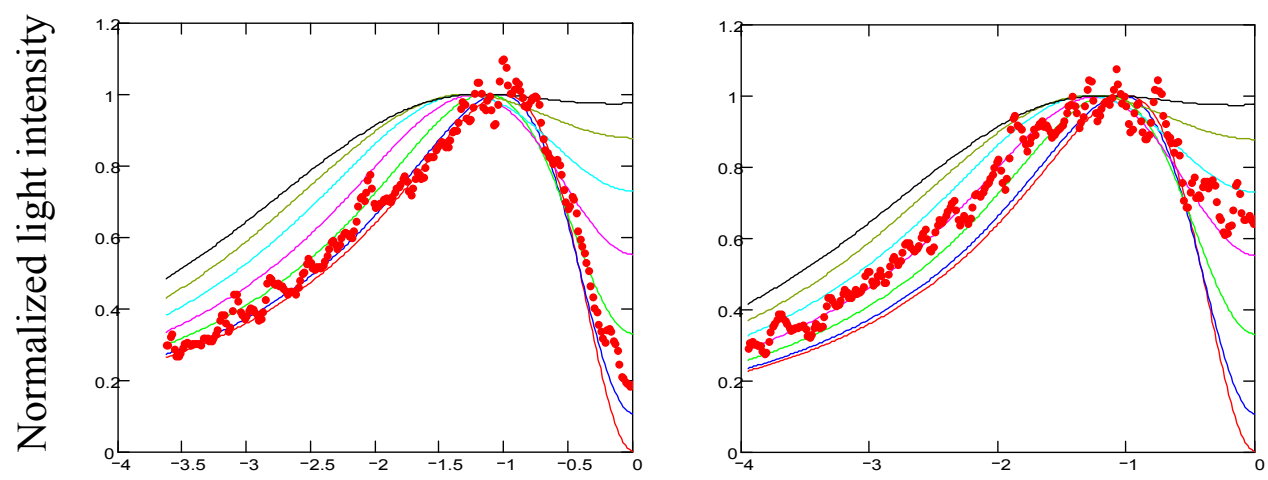


\section{Light collection angle in $1 / \gamma$ units}

Figure 13: Example OTR data fits to theoretical profiles

The value of the mean square error was calculated for each fit pattern, with the minimum value corresponding to the best divergence fit. A plot of the mean square error as a function of the divergence fit parameter is shown in Fig. 14. A parabolic fit to the mean square error in the vicinity of the best fit is used to determine the $\chi^{2}$ parameter for the analysis of each angular OTR pattern.

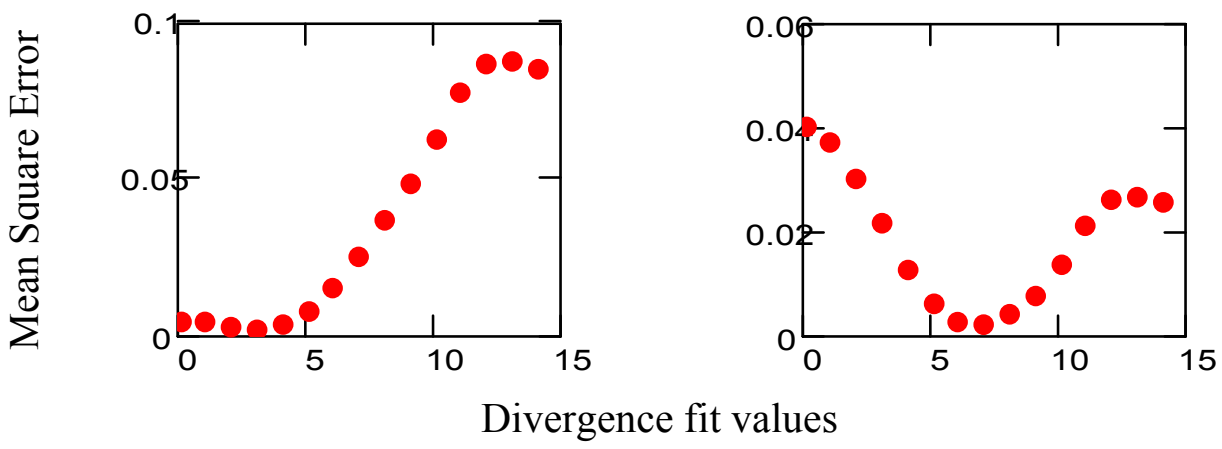

Figure 14: Least-squares fitting examples of OTR data

As described in the Sec. 1, measurement of beam divergence using OTR is limited at very high and very low divergence values. A low divergence or "cold" beam will produce an OTR pattern indistinguishable from the ideal single electron OTR pattern. A high divergence beam will wash out the lobe structure and fill in the null in the specular direction. To illustrate the useful range of divergence fits, the mean square error is plotted in Fig. 15 between neighboring fit curves $(2.5 \mathrm{mr}$ versus $0 \mathrm{mr}, 5.0 \mathrm{mr}$ versus 2.5 $\mathrm{mr}$, through $32.5 \mathrm{mr}$ versus $30 \mathrm{mr}$ ). A large mean square error is desirable between neighboring divergence fit values because that indicates that the data sets will be clearly distinguishable from each other. This effect is also apparent at the extreme values of divergence plotted for the data fits in Fig. 14. At $17.5 \mathrm{MeV}, 1 / \gamma$ corresponds to a $28.4 \mathrm{mr}$ divergence. Single-foil OTR measurements were limited in our case to the divergence range of approximately $26 \%$ to $95 \%$ of $1 / \gamma$ since the mean square error drops by a factor of two from its peak value at these divergences.

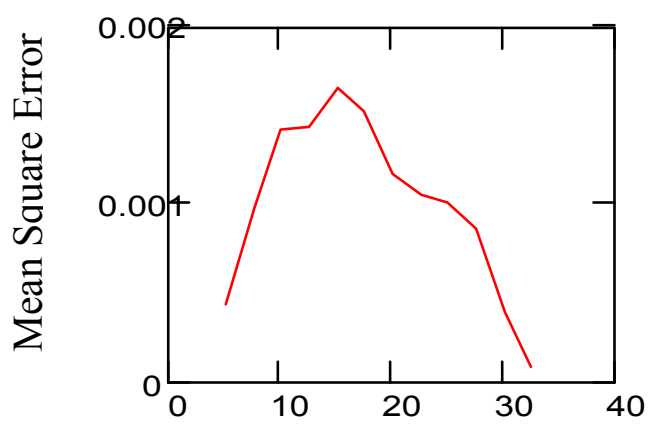




\section{Divergence (mr)}

Figure 15: Mean square error between nearest neighbor fits

Measuring a large angular field of view was one key requirement for our optical collection system. To illustrate the requirement for a wide angular field of view, the mean square error between data fits for 15 and $17.5 \mathrm{mr}$ is shown in Fig. 16 as a function of the angular extent of light collection. Collection of the OTR pattern past an angle of $\pm 4 / \gamma$ gives limited benefit since the mean square error asymptotes to a constant value, while the requirement to collect a significant fraction of the $\pm 4 / \gamma$ is also apparent. The full field of view in the FXR experiment was $\pm 4.1 / \gamma$. The impact of only using one OTR lobe was to double the uncertainty contribution of the angular data fitting.

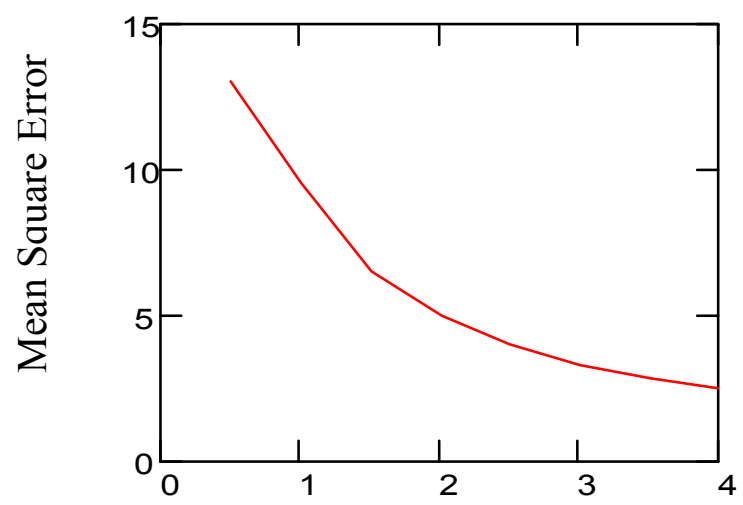

Extent of angular field of view (units of $1 / \gamma$ )

Figure 16: Mean square error for a $15 \mathrm{mr}$ fit as a function of collection angle

The measured rms divergence, rms beam radius, and normalized rms emittance for three time-steps near the center of the FXR beam pulse flat-top are shown in Fig. 17. The center of the flat-top corresponds to $80 \mathrm{~ns}$ camera trigger delay. Using $20 \mathrm{~ns}$ steps between data points, the emittance values were $10.7 \pm 1.3,8.6 \pm 1.0$, and $9.1 \pm 2.1 \mathrm{~cm}-\mathrm{mr}$ respectively. The emittance values for the time steps plotted in Fig. 17 are the product of the beam divergence and spot size at the OTR target foil. These emittance values are not normalized by $\beta \gamma$, which for $17.5 \mathrm{MeV}$ is equal to 35.23 . These data were collected with a $1 / 2$ ns gate width. The beam radii corresponding to these time-steps were measured as $5.00 \pm 0.59 \mathrm{~mm}, 5.12 \pm 0.59 \mathrm{~mm}$, and $2.61 \pm 0.60 \mathrm{~mm}$ respectively. The uncertainty in beam radius is dominated by the resolution of the optical system. Since the beam radius was small in the last time-step, the uncertainty introduced by the optical system generated a relatively large error bar. The curve in Fig. 17 illustrates the variation of divergence and beam radius quantitatively, and also demonstrates the consistency of the emittance during these variations within the statistical significance of the measurements. 


\section{Emittance versus time}

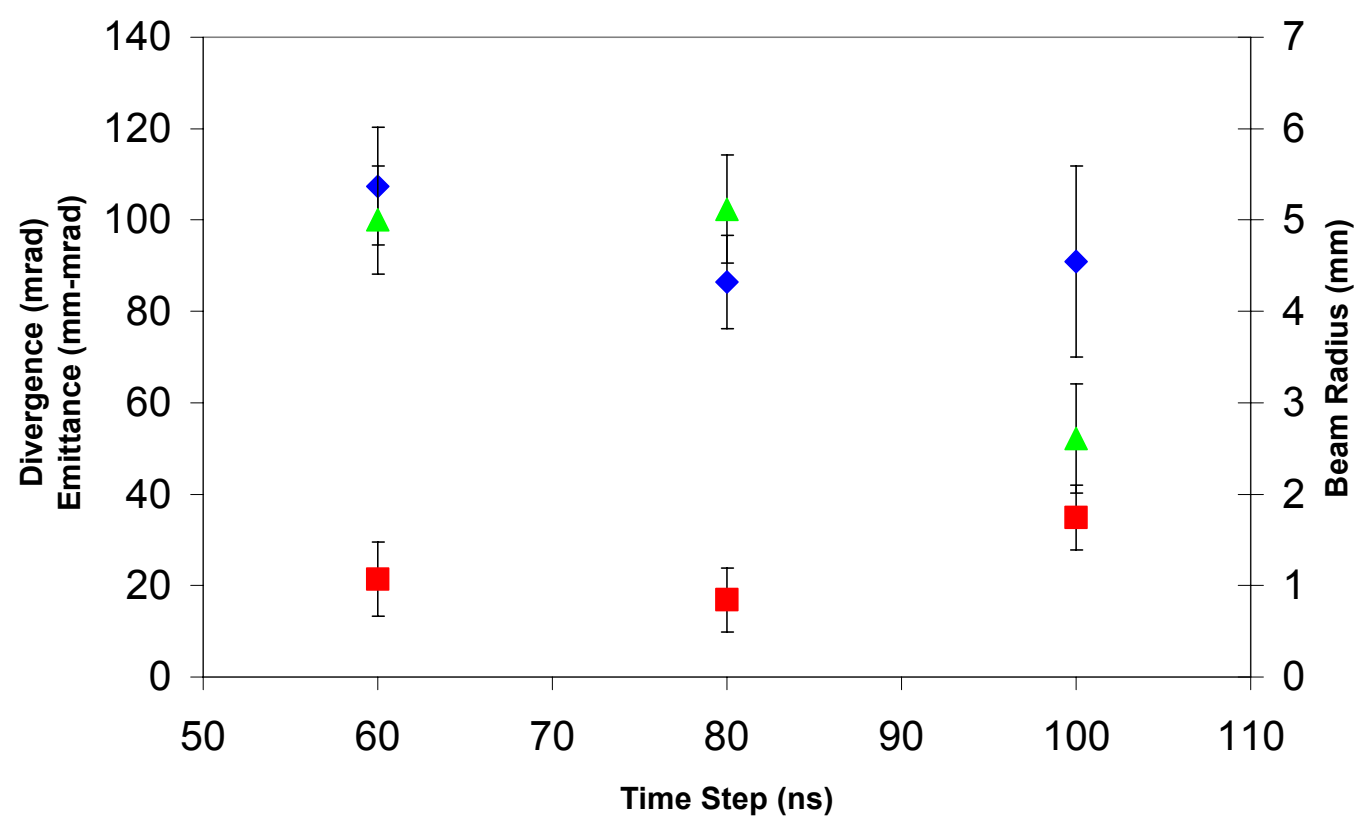

Figure 17: Divergence (red square), beam radius (green triangle), and emittance (blue diamond) versus time

\section{NEW OTR PROFILING TECHNIQUE}

One remarkable characteristic of OTR light is that it is radially polarized: the polarization vector lies in the plane formed by the normal to the OTR interface surface and the direction of the emitted photon. As described in the introduction, the observation plane for OTR light is defined by the direction of an emitted OTR photon and the normal to the OTR interface. Using a linear polarizer, orthogonal beam divergence characteristics can be measured by separating the OTR light that is parallel to the plane of observation. Orthogonal planes of observation can be used to measure the respective beam divergence in those orthogonal planes. This polarization characteristic was used to develop a new OTR measurement technique.

The Fresnel reflection coefficients of materials depend on incident angle, polarization, and wavelength. As mentioned previously, our OTR target was Aluminized Kapton. Aluminum and Kapton have very different reflection characteristics, especially with respect to their polarization dependence. Aluminum has reflection coefficients (absolute magnitude) of 0.929 and 0.863 for incident radiation with polarization parallel and perpendicular to the interface respectively [13]. These values correspond to 45 degree reflection at $500 \mathrm{~nm}$ wavelength. The values of the same reflection coefficients for Kapton are 0.146 and 0.021 [14]. Clearly, Kapton will reflect less of the OTR light than Aluminum for all polarizations, but it preferentially reflects light that is polarized parallel 
to the OTR interface (vertically polarized). A pure Kapton interface produces a reflected OTR pattern that appears to have been passed through a linear polarizer due to the large ratio between the reflection coefficients $\Gamma_{\text {parallel }}$ and $\Gamma_{\text {perpendicular. }}$ This effect does not occur to significant extent using Aluminum as the reflector.

With these facts in mind, we fabricated an OTR target that had aluminized regions on a Kapton substrate. Three circular regions were Aluminized, each with different diameters as shown in Fig. 18. The smallest circle was spatially smaller than the electron beam profile. Since OTR light will preferentially be reflected into the collection system from the Aluminized regions, especially in the case of polarization that is not parallel to the Kapton interface, the divergence of a small region of the beam profile can be examined. The Aluminized target diameters were $20 \mathrm{~mm}, 10 \mathrm{~mm}$, and $5 \mathrm{~mm}$.

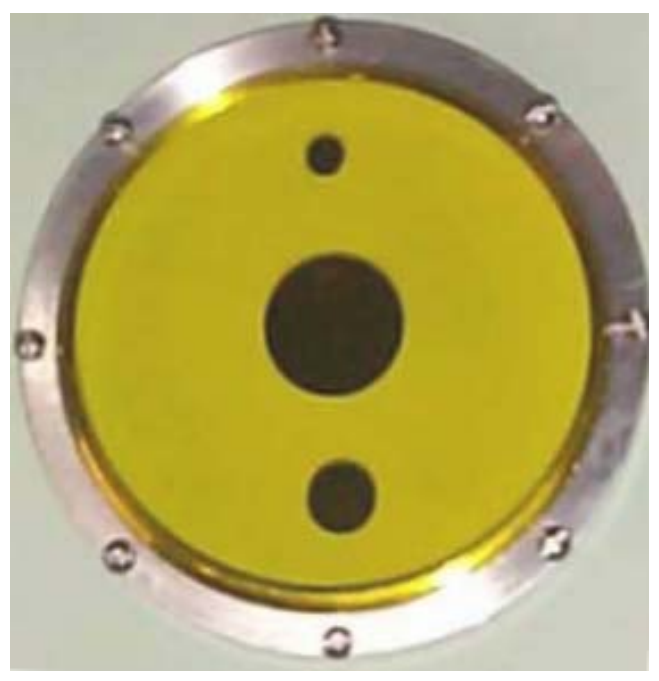

Figure 18: Mounted Kapton foil target with circular Aluminized regions

In addition to providing spatially resolved divergence measurement, a circular, aluminized region on a transparent background proved very useful as a means of spatial calibration. The $20 \mathrm{~mm}$ diameter Aluminum target illuminated by the $\mathrm{HeNe}$ alignment laser is shown in Fig. 19. With a known target size, we can easily calibrate the spatial magnification of the entire optical system. We can also verify the foil tilt angle by the aspect ratio of the width and height of the target image. The total field of view of the optical collection system in the imaging mode was also clear in this image, and has been emphasized with a dashed, white line in the figure. We were able in this way to additionally verify the range of our field of view and understand the range of spatial beam sizes that we could measure. Lastly, the edges of the Aluminized target provide an easy means of optimizing the focus of the imaging system. 


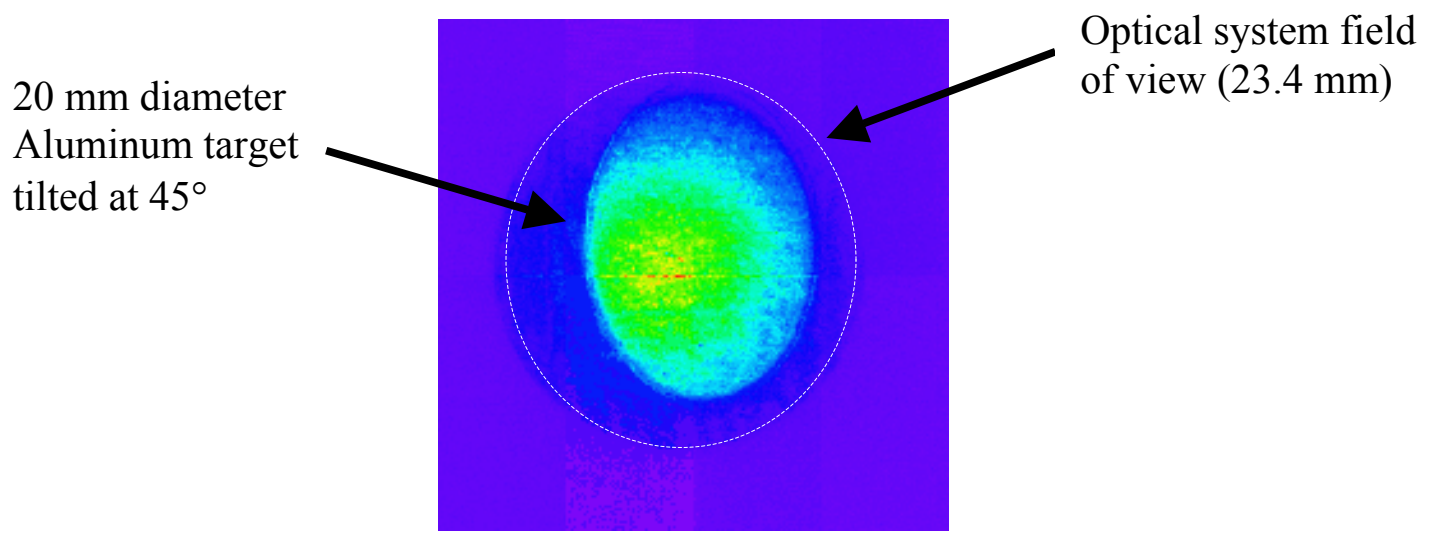

Figure 19: Alignment laser iluminates the $20 \mathrm{~mm}$ Aluminum target

To demonstrate the capability of the partially Aluminized Kapton target foil, the linear polarizer used in the optical collection system was turned successively in the vertical and horizontal directions. The $5 \mathrm{~mm}$ foil target is shown for both polarization cases in Fig. 20. As expected, in the vertical polarization case we measured a bright region representing the Aluminized target with a background of the full beam profile. In the case of horizontal polarization, only the Aluminized target region emits OTR light, while the light from the remainder of the beam profile is transmitted through the Kapton foil.

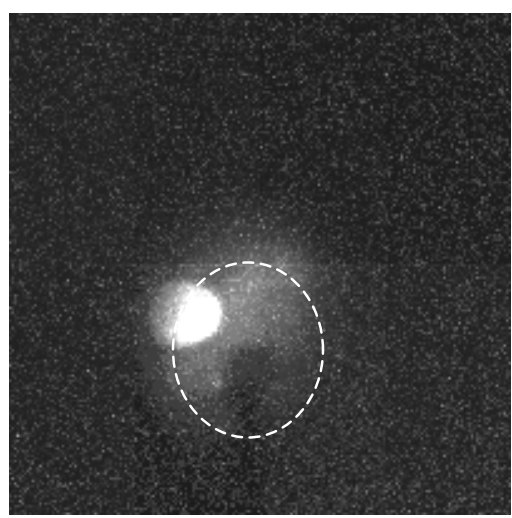

Vertical Polarization

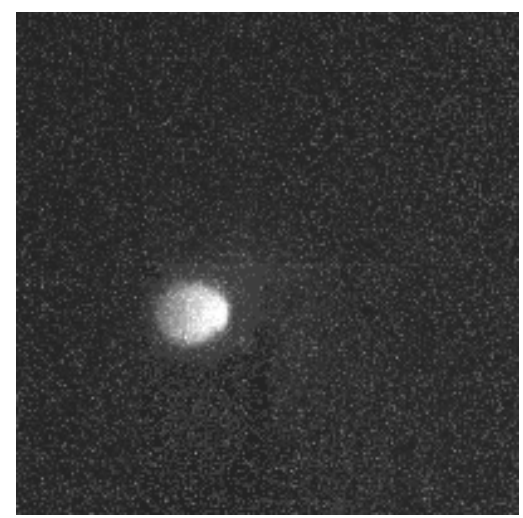

Horizontal Polarization

Figure 20: Aluminum target diameter of $5 \mathrm{~mm}$ on a Kapton substrate

The profile of whole beam is highlighted in the vertically polarized pattern with a dashed line. The dark region in beam profile in this case was due to a damage spot on the foil. The divergence fit of the angular pattern produced by the $5 \mathrm{~mm}$ foil target represented the smallest recorded value. That result is consistent with a phase-space diagram point of view. As shown in Fig. 21, we effectively sliced out and measured the divergence of a small region of the phase ellipse near its outer edge. 


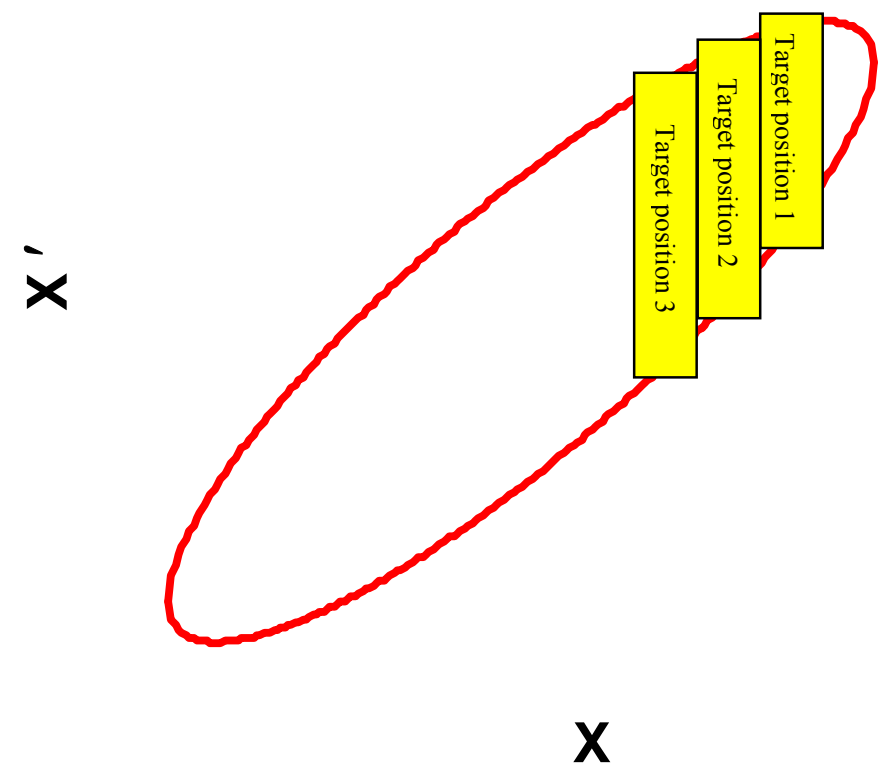

Figure 21: Schematic of spatial resolution within the beam phase-space

A foil target that is small in comparison to the beam profile can be scanned across the beam to give the divergence as a function of spatial coordinates. In this way the full phase ellipse of the beam can be analyzed. This same concept was demonstrated previously [11] by collecting the OTR light from the entire beam, imaging that light to a point external to the beam-foil interaction region, and masking part of the measured profile. The above technique represents a simplified version of this concept appropriate for a beam with a large spatial extent. Both versions of the OTR phase-space mapping idea additionally provide the important capability of measuring the tilt of the phase ellipse, verifying the beam waist condition at the foil. The optical imaging version of the OTR phase space mapping technique is currently also being extended to allow single-shot characterization of the full phase-space ellipse [17].

Two phase-space ellipses are shown in Fig. 22. The blue ellipse represents a beam with a large emittance focused to a waist. The red ellipse represents a diverging beam with half the emittance of the first case. The beam radius and divergence values based on OTR measurements will be the same for both cases, illustrating the importance of verifying the beam waist condition at the point of measurement. The technique of mapping beam divergence correlated to the position within the beam solves this problem since the ellipse tilt angle (envelope divergence) can be measured directly. This technique has been demonstrated using optical techniques [11], and can equivalently be achieved using the present scheme. 


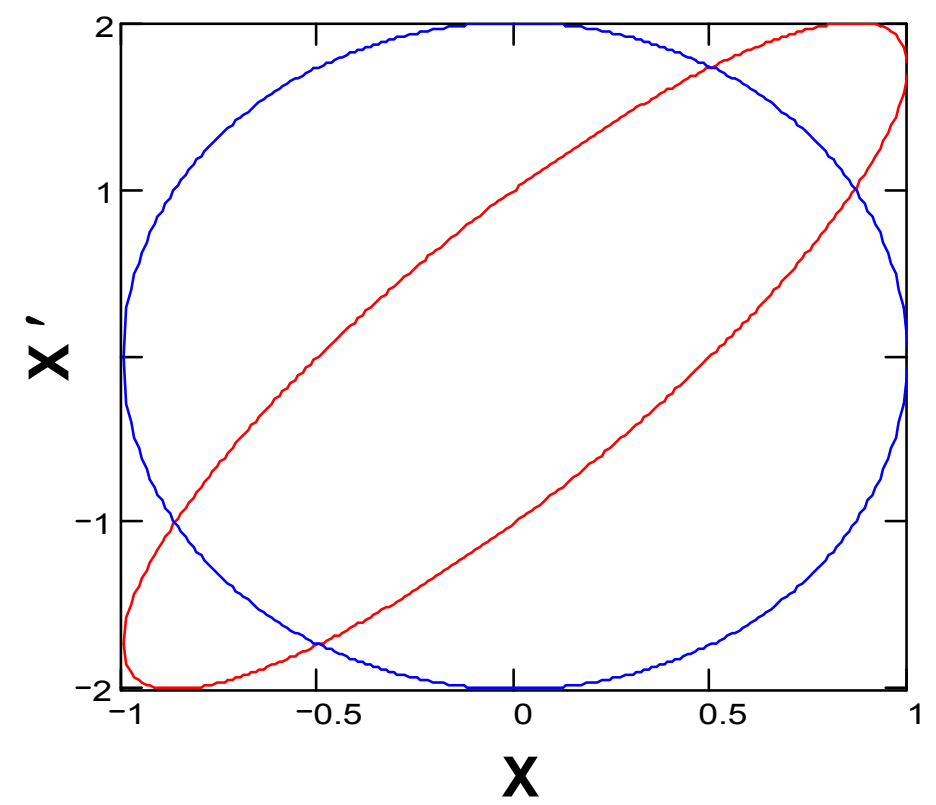

Figure 22: Beam envelope emittance uncertainty illustration

\section{CONCLUSIONS}

The divergence and radius of the FXR beam were characterized at various time steps using Optical Transition Radiation, allowing calculation of the rms emittance of the beam as a function of time. A new technique was also demonstrated that will allow spatially resolved characterization of the beam divergence, and direct measurement of the phasespace ellipse.

From the results of the measurement series, we may conclude that the emittance is relatively constant with time, but that the divergence and radius vary significantly. Since the transport system parameters are constant on the time-scale of the measurement, we may conclude that the divergence changes due to energy or current variations through the pulse. Since the consistency of the current is verified independently through beam current monitors, we may additionally conclude that the divergence and beam radius variations are most likely due to variations in beam energy as a function of time. The significant observation in this case is that even if the emittance is acceptable and constant, a beam with changing divergence and radius will not focus well to a final small spot.

\section{FUTURE PLANS}

The next logical step for the OTR emittance diagnostic is engineering a system that is more robust, flexible, and easy to operate. Alignment of the present system is time consuming, and the lens arrangement is vulnerable to being bumped and misaligned. A simpler system for recording the angular profile of an OTR pattern that includes a single lens and a diffusing screen has been demonstrated [12]. Using a large diffusing screen in 
the plane spaced at the exact focal length of the lens allows a spatially recording camera to capture the angular map from the diffusing screen.

Applying our optical system optimization techniques, we have improved the performance of the lens and screen system using two matched doublet lenses in place of a single lens to minimize the spherical aberration of the system. A schematic of the simplified optical collection system is shown in Fig. 23. The red, green and blue rays show three different angles that map to three points on the diffusing screen. The overall length from the foil target to the diffuser is $488 \mathrm{~mm}$. Since a relatively short section extends outside of the vacuum system, we plan to enclose the lenses and screen in a solid housing, affixed to the vacuum window flange. This will protect the optical collection system from accidental misalignment.

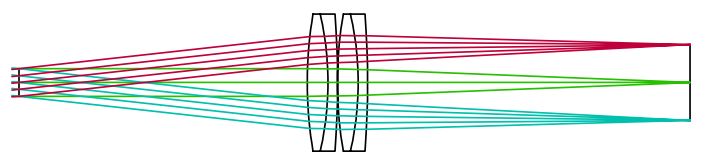

Figure 23: Schematic of proposed new OTR diagnostic arrangement

As shown in Fig. 24, the rms radius at the diffusing screen for a given angle is about 26 microns across the full field of the OTR light source, representing a significant improvement over the current system. The resolution limit for the diffusing screen may limit the ultimate system resolution, but we can replace it with a fiber optic faceplate with element sizes in the range of $6 \mu \mathrm{m}$ if necessary.

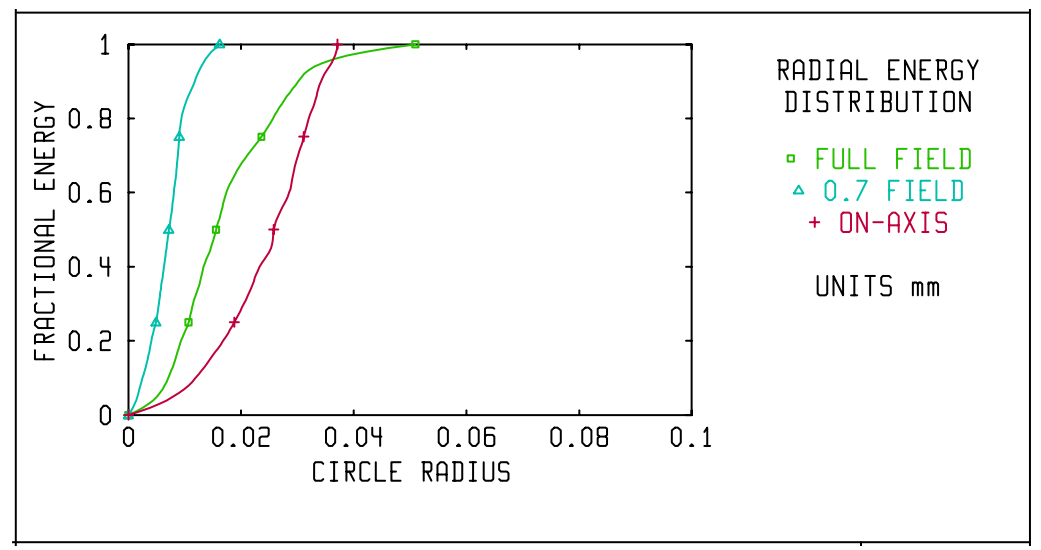

Figure 24: Optical performance of proposed OTR angular collection system

Since the described optical system is intended only for measurement of the angular OTR profile, a means of spatial beam characterization is still required. A schematic of a twocamera system is shown in Fig. 25. A new OTR target with a smooth Aluminized front face and an etched Quartz substrate will be used to produce OTR light from the front face, and diffused Cerenkov light from the rear face of the target for spatial characterization. Two co-timed gated cameras will be required for this new arrangement. The new system will additionally eliminate the uncertainty of shot-to-shot variations 
between collection of the angular and spatial profiles since both will be collected simultaneously.

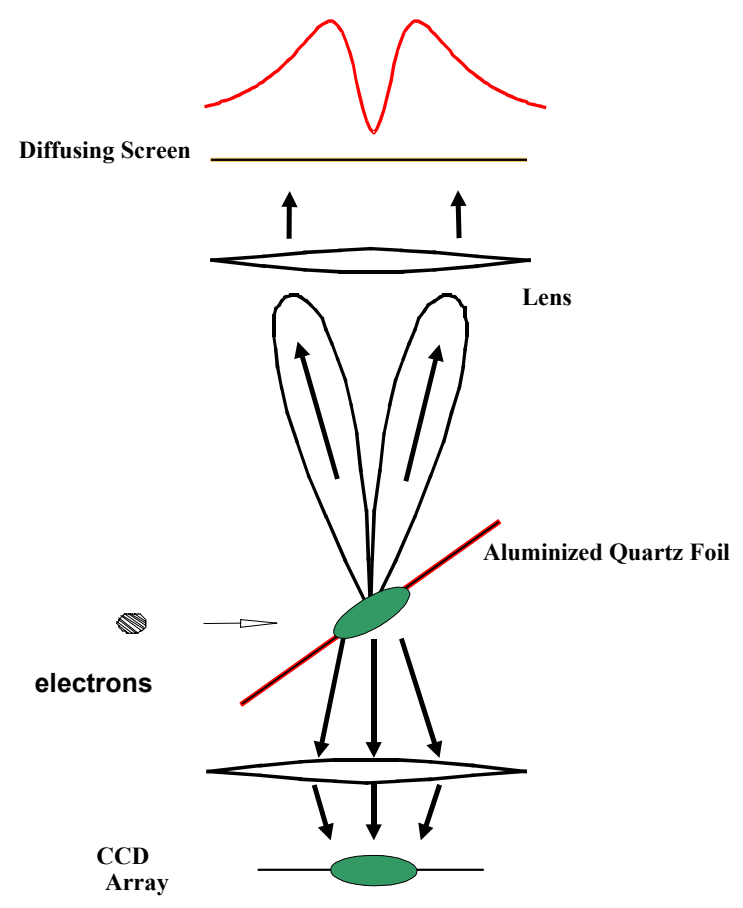

Figure 25: Simultaneous measurement of spatial and angular beam profiles

The imaging field of view was limited to $23.4 \mathrm{~mm}$ for the optical collection system in the spatial imaging mode. The new system that splits the imaging and divergence measurement tasks will additionally allow the spatial imaging system to collect a significantly wider field of view since the optics for this part of the system can be designed with the sole purpose of high resolution spatial imaging.

The next significant experiment planned using OTR is measurement of energy and energy variation of the FXR beam. Although the single-foil OTR lobe spacing is energy dependent, the sensitivity of the lobe spacing is not appropriate for energy resolution below the $10 \%$ energy variation range, even without considering beam divergence blurring. Using two closely spaced OTR target foils allows finer resolution of both beam divergence and energy variations. Multiple foils spaced appropriately for a given beam energy produce an OTR interference pattern (OTRI) that is spatially coherent [8]. A plot of a two-foil OTRI and single-foil OTR pattern for three energy values is shown in Fig. 26. From these figures, one can clearly see that we cannot effectively employ a single foil to look at energy variation, but that we can certainly use OTRI to diagnose beam energy within a $10 \%$ range by examining the innermost interference fringes. The foil spacing for these examples is $1.5 \mathrm{~mm}$. 

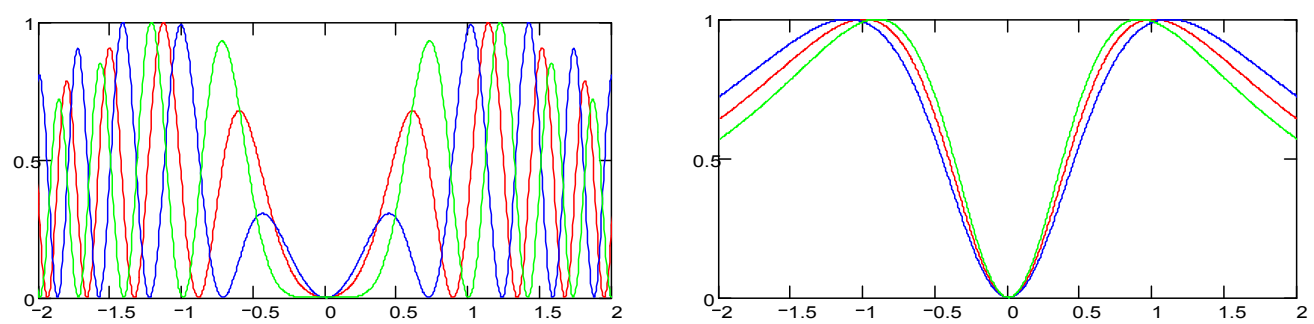

Figure 26: OTR and OTRI patterns for $17.5 \mathrm{MeV} \pm 10 \%$

The large spatial extent of the FXR beam will not allow the OTRI light to escape between the two foils of the interferometer, necessitating an OTR interferometer with a clear front foil. For higher energy applications corresponding to larger foil spacing, metalized foils can be used for an OTR interferometer. This arrangement simplifies the collection of the OTRI pattern since the incoherent light from the front surface of the first foil can be discarded. In the present case with closely spaced, transparent foils, we need to account both for the spatially coherent OTRI, and the OTR from the front foil added together [9]. The three OTR contributions to the total measured OTRI pattern for the planned FXR experiment are shown schematically in Fig. 27.

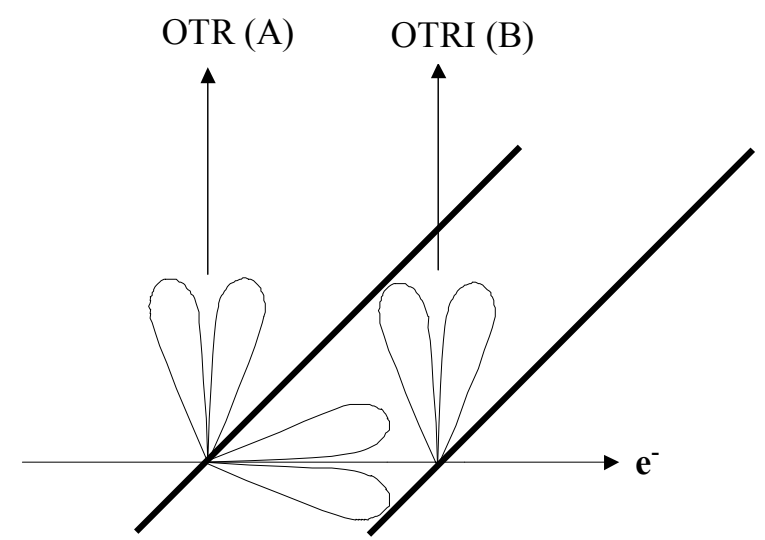

Figure 27: Clear front foil OTRI arrangement

In order to analyze the expected OTR patterns from this type of interferometer, we combine the single-foil (incoherent forward coefficient $A$ ' and reflected coefficient $A$ ) and two-foil expressions (coherent coefficient $B$ ) to give the following form

$\frac{\partial^{2} I_{\|}}{\partial \omega \partial \Omega}=\sum_{m}\left(A+A^{\prime}\right) \cdot \frac{\left(\theta-\theta_{m}\right)^{2}}{\left(\gamma^{-2}+\left(\theta-\theta_{m}\right)^{2}\right)^{2}}+B \cdot \frac{\left(\theta-\theta_{m}\right)^{2}}{\left(\gamma^{-2}+\left(\theta-\theta_{m}\right)^{2}\right)^{2}} \cdot \sin ^{2}\left\{\frac{\pi \cdot L}{2 \cdot \lambda_{0}}\left[\frac{1}{\gamma^{2}}+\left(\theta-\theta_{m}\right)^{2}\right]\right\}$

Since finely spaced lobes will not be resolvable for the FXR OTR beam, and since the OTRI goal in this case is energy measurement, a spacing of $0.5 \mathrm{~mm}$ was examined. In this case, the lobe heights are less sensitive to divergence, but the few lobes near the center are more distinct from the background. The expected OTRI patterns for a clearfoil OTR interferometer with a $0.5 \mathrm{~mm}$ foil spacing, a $17.5 \mathrm{MeV}$ beam energy, and $5 \mathrm{mr}$ 
divergence is shown in Fig. 28. This divergence will require a large spatial beam size at the foil (rms radius of $\sim 17 \mathrm{~mm}$ ), but again since we are not interested in collecting divergence information in this case, losing light from part of the beam is not important unless there are correlations between energy spread and transverse spatial coordinates within the beam. Energy variations on the order of $1 \%$ should be resolvable using a twofoil interferometer with these specifications. The lobe structure of the OTRI is washed out at the $10 \mathrm{mr}$ divergence range. The OTRI pattern with no divergence is shown in the figure in light blue, and corresponds to the curve with the sharpest interference fringes. These example interference patterns assume an optical wavelength of $500 \mathrm{~nm}$. In practice, an appropriate optical filter will be necessary to reduce fringe blurring due to finite optical bandwidth effects as well. Energy spread can also blur the OTRI fringes. This effect should be minimized when examining a short time slice in the beam, though we will also be able to characterize this effect as a function of gate width.
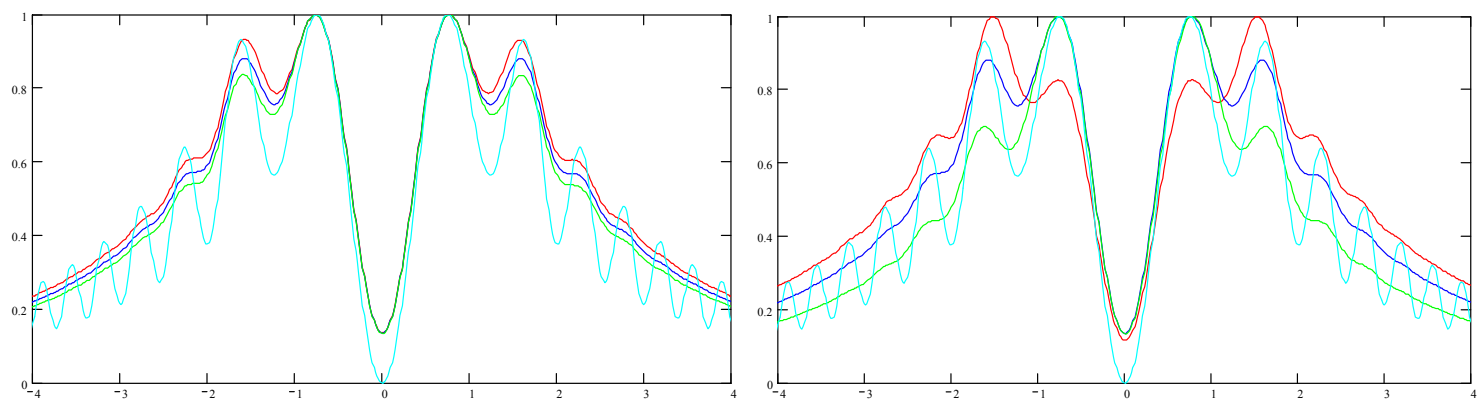

Figure 28: OTRI patterns for $17.5 \mathrm{MeV} \pm 2 \%$ and $\pm 10 \%$ with $5 \mathrm{mr}$ divergence

Given the expected range of divergence for the FXR electron beam, the two-foil OTRI diagnostic will have limited usefulness as an emittance diagnostic. The effective measurement range for a two-foil interferometer is approximately $13 \%$ to $25 \%$ of $1 / \gamma$, covering the range of lower divergence beam applications. In this case, the lower limit of divergence resolution is due to scattering in the first foil. For a $17.5 \mathrm{MeV}$ beam going through $6.7 \mu \mathrm{m}$ of Kapton, the scattering angle is $3.6 \mathrm{mr}$, or $12.7 \%$ of $1 / \gamma$. At $15-20 \mathrm{mr}$ divergence $(53 \%-56 \%$ of $1 / \gamma)$, the fine fringes outside of the $2 / \gamma$ range (past the first $2-3$ fringes) are completely washed out. Since the innermost lobes of the OTRI pattern are most sensitive to energy variation, the divergence limitation does not preclude the usefulness of OTRI as an energy diagnostic. An OTR interferometer can be used to quantify both the beam energy, and its variation through the pulse. Since the inner lobes provide the most sensitivity to energy variation, we may also limit the angular range collected to increase angular resolution.

The front foil also needs to be carefully chosen for two additional reasons. If the foil is too thin, coherence of light from front and back of first foil can cause peaks in the optical pattern that will confuse the energy measurement result. We may need to use a thinner foil target in order to limit the beam divergence in the OTR interferometer. If we measure OTRI light over a wavelength range of 500 to $600 \mathrm{~nm}$, the foil thickness is on the order of 17 wavelengths, and should not cause optical coherence effects. 
The OTRI energy diagnostic should also prove useful as a reasonably sensitive central energy measurement. As was demonstrated in the DARHT OTR experiments [12], we have the possibility of using a streak camera and slit to get a continuous measurement of the OTR pattern produced by the beam. In our case, we can apply this technique to characterize energy as a continuous function of time. Since the spatial movement of the beam spot will not affect the collection of the angular OTR pattern, the slit imaging technique should work for a streaked energy measurement despite any transverse beam movement during the pulse.

\section{Acknowledgments}

Work performed under the auspices of the U.S. Dept. of Energy by the Lawrence Livermore National Laboratory under Contract W-7405-Eng-48.

I want to recognize and thank Mike Ong and Paul Wargo for providing the framing camera and fiber optic transport to the diagnostic, as well as providing the system for data collection and recording. I also want to thank Lynn Seppala for optics design. Christophe Vermare and David Mohr at the LANL DARHT facility helped with important advice for the OTR experiment. Don Rule provided advice and information regarding the optical properties of target materials. I would also like to thank the FXR staff for accelerator operation and successful implementation and execution of the experiment. That group included Jan Zentler, Glen Westenskow, Mike Mcgregor, Jim Dunlap, and Marcus Libkind.

\section{References}

[1] R. Scarpetti, J. Zentler, J. Boyd, G. Earley, R. Kerr, R. Kihara, K. Griffin, "Upgrades to the LLNL Flash X-Ray Induction Linear Accelerator (FXR)," $11^{\text {th }}$ IEEE International Pulsed Power Conference, Baltimore, MD, June 29-July 2, 1997

[2] G.P. Le Sage, S.G. Anderson, T.E. Cowan, J.K. Crane, T. Ditmire, J.B. Rosenzweig, "RF photoinjector development for a short-pulse, hard X-ray Thomson scattering source," AIP Conference Proceedings, (no 569), p.391-404, 2001

[3] S. Humphries, Charged Particle Beams, John Wiley and Sons, Inc., 1990

[4] J. Rosenzweig, G. Travish, "Design considerations for the UCLA PBPL slit-based phase space measurement system," March 2, 1994, Technical note on-line http://pbpl.physics.ucla.edu/papers

[5] H. Wiedemann, Particle accelerator physics: basic principles and linear beam dynamics, Springer-Verlag, New York, 1993.

[6] W.E. Nexsen, R.D. Scarpetti, J. Zentler, "Reconstruction of FXR Beam Conditions," Particle Accelerator Conference, 2000 
[7] V. L. Ginsburg and I.M. Frank, JETP (USSR) 16 (1946) 15

[8] L. Wartski et al., J. Appl. Phys. 46 (1975) 3644

[9] Ralph B. Fiorito and Donald W. Rule, "Optical transition radiation beam emittance diagnostics," in: Beam Instrumentation Workshop, ed. R.E. Shafer, (AIP Conf. Proc. No. 319, AIP Press, 1994).

[10] D.W. Rule, "Transition radiation diagnostics for intense charged particle beams," Nucl. Instrum. Meth. B24/25, 901 (1987).

[11] "Transverse phase space mapping of relativistic electron beams using Optical Transition Radiation," G. P. Le Sage, T. E. Cowan, R. B. Fiorito, and D. W. Rule, Phys. Rev. ST Accel. Beams 2, December 1999.

[12] C. Vermare and D.C. Moir , "Angular distribution measurement of an Intense Pulsed Electron Beam using the Optical Transition Radiation (OTR)", LA-UR-01-1171, March 6,2001

[13] Handbook of optical constants of solids, Edward D. Palik Editor, Academic Press, 2 volumes ISBN 0-12-544422-2

[14] "The optical properties of Kapton: Measurement and applications", E.T. Arakawa, M.W. Williams, J.C. Ashley, and L.R. Painter, J. Appl. Phys. 52, 3579 (1981)

[15] E. Hecht, Optics, Addison Wesley Pub. Co., 1974

[16] H.A. Bethe and J. Ashkin, Experimental Nuclear Physics, Vol. 1, ed. E. Segre, Wiley, New York, 1953

[17] Transverse phase space mapping of relativistic beams using optical transition radiation, R.B. Fiorito, DOE SBIR 2001, Technical Topics Section 4b 
University of California

Lawrence Livermore National Laboratory

Technical Information Department

Livermore, CA 94551

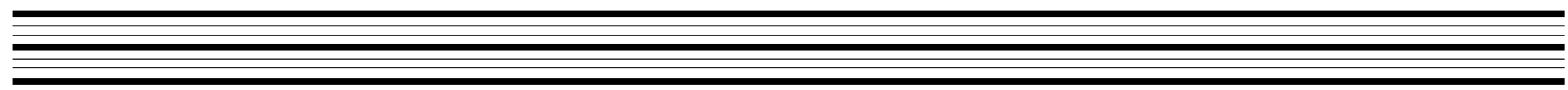

